\title{
Multiple Adaptive Fading Cubature Kalman Filter for INS/GPS Integrated Navigation
}

\author{
Wei Lin \\ China North Industries Corp., Beijing 100053, China \\ linwei@norinco.cn (Corresponding Author)
}

\begin{abstract}
Keywords:INS/GPS; integrated navigation; CubatureKalmanFilter; multiple fading factor.
Abstract.A multiple adaptive fading cubature Kalman filter (MAFCKF) is designed by introducing the multiple fading factors to mitigate the negative effects of the uncertainties in dynamics or measurement model. The effectiveness of the proposed MAFCKF was demonstrated and proved by the INS/GPS integrated navigation simulations.
\end{abstract}

\section{Introduction}

Inertial Navigation System(INS) and Global Positioning System (GPS) integrated navigation system has been used in many fields, such as aircraft, missile and unmanned aerial vehicle [1]. The INS/GPS integrated navigation is often realized using the Kalman filter to estimate the host platform attitude. However, if the system parameters which are used to update the state and covariance estimates are not accurately modeled, the accuracy of the state estimates may significantly degrade.Fortunately, a single adaptive fading factor can be introduced as a multiplier to the dynamic or measurement noise covariance to adjust the priori covariance when the information of the dynamic or measurement model is incomplete [2-5]. Then, considering the complex systems with multivariable, the multiple fading factor is proposed to reflect corrective effects of the multivariable in filtering[2,5]. For the nonlinear of the INS/GPS integrated navigation, the cubature Kalmanfilter (CKF) [6, 7] is introduced to avoid calculating Jacobians or Hessiansas the extended Kalman filter[1]. This papermainly focuses onproposing a multiple adaptive fading cubatureKalman filter (MAFCKF)for integrated navigation system with inaccuratemodels.

\section{INS/GPS Integrated Navigation System}

For the INS/GPS Integrated Navigation System, the specific forces obtained by the INSwhich consists ofgyroscopes and accelerometers, and the navigation parameters measured by the GPS, are loaded into the filter to get the optimal estimations of the navigation parameters and attitude errors. TheINS navigation coordinate system isthe East-North-Up (ENU) frame, and the attitude error equationsandarrangeequations of the INS system can be found in [1]. The measurement model of the INS/GPS integrated navigation system is established using the position and velocity information of the GPS, and the measurement equations are

$$
z=\left[v_{E G}, v_{N G}, v_{U G}, L_{G}, \lambda_{G}, h_{G}\right]^{T}
$$

where $v_{E G}, v_{N G}, v_{U G}$ are the velocities of the GPS, and $L_{G}, \lambda_{G}, h_{G}$ are the position information of the GPS.

The state of the INS/GPSintegrated navigation system is described as

$$
\boldsymbol{x}=\left[\phi_{E}, \phi_{N}, \phi_{U}, v_{E}, v_{N}, v_{U}, L, \lambda, h, \varepsilon_{b E}, \varepsilon_{b N}, \varepsilon_{b U}\right]^{T}
$$

and the state equation is given by

$$
\boldsymbol{x}(t)=\boldsymbol{f}(\boldsymbol{x}(t), \boldsymbol{w}(t), t)
$$

The corresponding measurement equation is

$$
\boldsymbol{z}(t)=\boldsymbol{H} \boldsymbol{x}(t)+\boldsymbol{v}(t)
$$

where $\boldsymbol{H}$ is a liner function, and its value is $\boldsymbol{H}=\left[\boldsymbol{I}_{6 \times 6}, \boldsymbol{O}_{6 \times 6}\right]$. 


\section{Multiple Adaptive Fading Cubature Kalman Filter Algorithm}

Consideringthe discrete nonlinear process and measurement models with additive noises given by

$$
\begin{gathered}
\boldsymbol{x}_{k}=\boldsymbol{f}\left(\boldsymbol{x}_{k-1}, \boldsymbol{u}_{k-1}\right)+\boldsymbol{w}_{k-1} \\
\boldsymbol{z}_{k}=\boldsymbol{h}\left(\boldsymbol{x}_{k}, \boldsymbol{u}_{k}\right)+\boldsymbol{v}_{k}
\end{gathered}
$$

where $\boldsymbol{x}_{k}$ is the $n \times 1$ state vector, $\boldsymbol{z}_{k}$ the $m \times 1$ measurement vector, $\boldsymbol{f}$ the dynamicvector-valued function, $\boldsymbol{h}$ the measurement vector-valued function. $\boldsymbol{u}_{k}$ the known control input vector. $\boldsymbol{w}_{k}$ and $\boldsymbol{v}_{k}$ are both independent zero-mean Gaussian noise processes, andtheir covariance are $\boldsymbol{Q}_{k}$ and $\boldsymbol{R}_{k}$, respectively.They satisfy

$$
E\left[\boldsymbol{w}_{k} \boldsymbol{w}_{j}^{\mathrm{T}}\right]=\boldsymbol{Q}_{k} \boldsymbol{\delta}_{i j}, E\left[\boldsymbol{v}_{k} \boldsymbol{v}_{j}^{\mathrm{T}}\right]=\boldsymbol{R}_{k} \boldsymbol{\delta}_{i j}, E\left[\boldsymbol{w}_{k} \boldsymbol{v}_{j}^{\mathrm{T}}\right]=\mathbf{0}
$$

where $\boldsymbol{Q}_{k}$ is positive semi-definite, and $\boldsymbol{R}_{k}$ is positive definite.

In the optimal Kalman filter, there is an orthogonal principle that the predicted residual sequence $\left\{\gamma_{k}\right\}$ is mutuallyorthogonal when the optimal gain matrix is calculated online [2]. The optimal gain matrix is obtained in the linear Kalman filter by minimizing the following equation

$$
E\left[\left(\boldsymbol{x}_{k}-\hat{\boldsymbol{x}}_{k}\right)\left(\boldsymbol{x}_{k}-\hat{\boldsymbol{x}}_{k}\right)^{T}\right], k=1,2, \mathrm{~L}
$$

and the following equation

$$
E\left[\gamma_{k+j} \gamma_{k}^{\mathrm{T}}\right]=0, k=1,2, \mathrm{~L}, j=1,2,3, \mathrm{~L}
$$

must be satisfied. In Eq. (9), the predicted residual vector can be defined by

$$
\boldsymbol{\gamma}_{k}=\boldsymbol{z}_{k}-\boldsymbol{H}_{k} \hat{\boldsymbol{x}}_{k / k-1}
$$

where $\boldsymbol{H}_{k}=\left.\frac{\partial \boldsymbol{h}\left(\boldsymbol{x}_{k}\right)}{\partial \boldsymbol{x}_{k}}\right|_{\boldsymbol{x}_{k}=\hat{x}_{k / k-1}}$.

To consider the incomplete information from the covariance of the states and noises, the multiple fading factors are inserted on the outside of the a priori error covariance. Based on the comment in the literature [5], a multiple fading factorwhich is premultiplicated to the priori error covariance equation for the linear Kalman filter are calculatedfor the CKF as follows. From the formula of the KF and the CKF, we have

$$
\boldsymbol{P}_{x z, k}^{a}=\boldsymbol{P}_{k / k-1}^{a} \boldsymbol{H}_{k}^{T}
$$

where $\boldsymbol{P}_{k / k-1}^{a}$ is symmetric and positivedefinite, and

$$
\boldsymbol{H}_{k}=\left(\boldsymbol{P}_{x z, k}^{a}\right)^{T}\left(\boldsymbol{P}_{k / k-1}^{a}\right)^{-1}
$$

Then,

$$
\boldsymbol{M}_{k}=\boldsymbol{P}_{x z, k}^{a}\left(\boldsymbol{P}_{x z, k}^{a}\right)^{T}\left(\boldsymbol{P}_{k / k-1}^{a}\right)^{-1}
$$

Finally, the multiple fading factors for the CKF can be calculated by the following equations

$$
\begin{gathered}
\lambda_{i, k}=\max \left\{1, \beta_{i} \tau_{k}\right\}, i=1,2, \mathrm{~L}, n \\
\tau_{k}=\frac{\operatorname{tr}\left[\boldsymbol{O}_{k}\right]}{\sum_{i=1}^{n} \beta_{i} \boldsymbol{M}_{i i, k}} \\
\boldsymbol{M}_{k}=\boldsymbol{P}_{x z, k}^{a}\left(\boldsymbol{P}_{x z, k}^{a}\right)^{T}\left(\boldsymbol{P}_{k / k-1}^{a}\right)^{-1} \\
\boldsymbol{O}_{k}=\boldsymbol{\Omega}_{k}-\varepsilon \boldsymbol{R}_{k} \\
\boldsymbol{\Omega}_{k+1}=\left\{\begin{array}{c}
\boldsymbol{\gamma}_{1} \boldsymbol{\gamma}_{1}^{\mathrm{T}}, \quad k=1 \\
\frac{\rho \boldsymbol{\Omega}_{k}+\boldsymbol{\gamma}_{k+1} \gamma_{k+1}^{\mathrm{T}}}{1+\rho}, \quad k>1
\end{array}\right.
\end{gathered}
$$

The cubature Kalman filter is a Bayesian filter under Gaussian assumption, which approximates the mean and covariance of a random variable by propagating under a nonlinear function following the cubature rule[6][8]. The algorithmic flow of theMAFCKF is givenas 
1) Initialize the state estimate $\hat{\boldsymbol{x}}_{\boldsymbol{0}}$ and covariance $\boldsymbol{P}_{\boldsymbol{0}}$.

2) Time update

(1)Generating the cubature points

$$
\chi_{j, k / k-1}=\sqrt{\boldsymbol{P}_{k-1}} \boldsymbol{\xi}_{j}+\hat{\boldsymbol{x}}_{k-1}, j=1,2, \mathrm{~L}, 2 n
$$

(2)Computingthe propagated cubature points through the nonlinear function

$$
\chi_{j, k / k-1}^{*}=\boldsymbol{f}\left(\chi_{j, k-1}, \overline{\boldsymbol{c}}, \boldsymbol{u}_{k-1}\right), j=1,2, \mathrm{~L}, 2 n
$$

(3) Computingthe prior state estimate

$$
\hat{\boldsymbol{x}}_{k / k-1}=\frac{1}{2 n} \sum_{j=1}^{2 n} \chi_{j, k / k-1}^{*}
$$

(4) Computing the multiple fading factors

$$
\begin{gathered}
\boldsymbol{P}_{k / k-1}^{a}=\frac{1}{2 n} \sum_{j=1}^{2 n} \chi_{j, k / k-1}^{*} \chi_{j, k / k-1}^{* T}-\hat{\boldsymbol{x}}_{k / k-1} \hat{\boldsymbol{x}}_{k / k-1}^{T}+\boldsymbol{Q}_{k-1} \\
\chi_{j, k / k-1}^{a}=\sqrt{\boldsymbol{P}_{k / k-1}^{a}} \boldsymbol{\xi}_{j}+\hat{\boldsymbol{x}}_{k / k-1}, j=1,2, \mathrm{~L}, 2 n \\
\boldsymbol{Z}_{j, k}^{a}=\boldsymbol{h}\left(\chi_{j, k / k-1}^{a}, \overline{\boldsymbol{c}}, \boldsymbol{u}_{k}\right), j=1,2, \mathrm{~L}, 2 n \\
\hat{\boldsymbol{z}}_{k}^{a}=\frac{1}{2 n} \sum_{j=1}^{2 n} \boldsymbol{Z}_{j, k}^{a} \\
\boldsymbol{P}_{x z, k}^{a}=\frac{1}{2 n} \sum_{j=1}^{2 n} \chi_{j, k / k-1}^{a} \boldsymbol{Z}_{j, k}^{a T}-\hat{\boldsymbol{x}}_{k / k-1} \hat{\boldsymbol{z}}_{k}^{a T}
\end{gathered}
$$

Substituting Eqs. (22)and(26) into Eq.(16) to calculate the fading factors, and the calculating formula are Eqs. (14)-(18).

(5) Computingthe prior error covariance

$$
\boldsymbol{P}_{k / k-1}=\boldsymbol{S}_{k}\left(\frac{1}{2 n} \sum_{j=1}^{2 n} \chi_{j, k / k-1}^{*} \chi_{j, k / k-1}^{* T}-\hat{\boldsymbol{x}}_{k / k-1} \hat{\boldsymbol{x}}_{k / k-1}^{T}+\boldsymbol{Q}_{k-1}\right)
$$

(6) Redrawing the cubature points using $\hat{\boldsymbol{x}}_{k / k-1}$ and $\boldsymbol{P}_{k / k-1}$

3) Measurement update

$$
\chi_{j, k / k-1}=\sqrt{\boldsymbol{P}_{k / k-1}} \boldsymbol{\xi}_{j}+\hat{\boldsymbol{x}}_{k / k-1}, j=1,2, \mathrm{~L}, 2 n
$$

(1) Computing the predicted measurement and corresponding covariance

$$
\begin{gathered}
\boldsymbol{Z}_{j, k}=\boldsymbol{h}\left(\chi_{j, k / k-1}, \overline{\boldsymbol{c}}, \boldsymbol{u}_{k}\right) \\
\hat{\boldsymbol{z}}_{k}=\frac{1}{2 n} \sum_{j=1}^{2 n} \boldsymbol{Z}_{j, k} \\
\boldsymbol{P}_{z z, k}=\frac{1}{2 n} \sum_{j=1}^{2 n} \boldsymbol{Z}_{j, k} \boldsymbol{Z}_{j, k}^{T}-\hat{\boldsymbol{z}}_{k} \hat{\boldsymbol{z}}_{k}^{T}+\boldsymbol{R}_{k} \\
\boldsymbol{P}_{x z, k}=\frac{1}{2 n} \sum_{j=1}^{2 n} \chi_{j, k / k-1} \boldsymbol{Z}_{j, k}^{T}-\hat{\boldsymbol{x}}_{k / k-1} \hat{\boldsymbol{z}}_{k}^{T}
\end{gathered}
$$

(2) Computing the filtering gain

$$
\boldsymbol{K}_{k}=\boldsymbol{P}_{x z, k}\left(\boldsymbol{P}_{z z, k}\right)^{-1}
$$

(3) Computing the a posterior estimation and the associated covariance

$$
\begin{gathered}
\hat{\boldsymbol{x}}_{k}=\hat{\boldsymbol{x}}_{k / k-1}+\boldsymbol{K}_{k}\left(\boldsymbol{z}_{k}-\hat{\boldsymbol{z}}_{k}\right) \\
\boldsymbol{P}_{k}=\boldsymbol{P}_{k / k-1}-\boldsymbol{P}_{x z, k} \boldsymbol{K}_{k}^{T}-\boldsymbol{K}_{k} \boldsymbol{P}_{x z, k}^{T}+\boldsymbol{K}_{k} \boldsymbol{P}_{z z, k} \boldsymbol{K}_{k}^{T}
\end{gathered}
$$




\section{Simulation and Results}

The initial attitudes, velocities and positions are assumed as $\left(120^{\circ} 32^{\prime}, 40^{\circ} 11^{\prime}, 1787.5 m\right)$, $(103.98 \mathrm{~m} / \mathrm{s}, 14.61 \mathrm{~m} / \mathrm{s}, 25.00 \mathrm{~m} / \mathrm{s})$ and $\left(12^{\circ} 00^{\prime}, 0^{\circ}, 0^{\circ}\right)$,the corresponding initial errors are $\left(1.5^{\prime}, 1.5^{\prime}, 50 \mathrm{~m}\right),(10 \mathrm{~m} / \mathrm{s}, 5 \mathrm{~m} / \mathrm{s}, 5 \mathrm{~m} / \mathrm{s})$ and $\left(50^{\prime}, 50^{\prime}, 50^{\prime}\right)$. The constant bias of the gyroscopes is $0.1 \%$, and the rate random walk of the gyroscopesis $0.05 \% \sqrt{h}$; the offset error of the accelerometer is $10^{-3} \mathrm{~g}$, and the rate random walk of the accelerometer is $10^{-4} \mathrm{~g}$. The position and velocity errors of GPS are $5 \mathrm{~m}$ and $0.1 \mathrm{~m} / \mathrm{s}$, respectively. The horizontal position error of the GPSis $0.02^{\prime}$, the vertical error $5 \mathrm{~m}$, and the velocity error $0.1 \mathrm{~m} / \mathrm{s}$. The reference trajectory of one aircraft is shown in Fig.1.

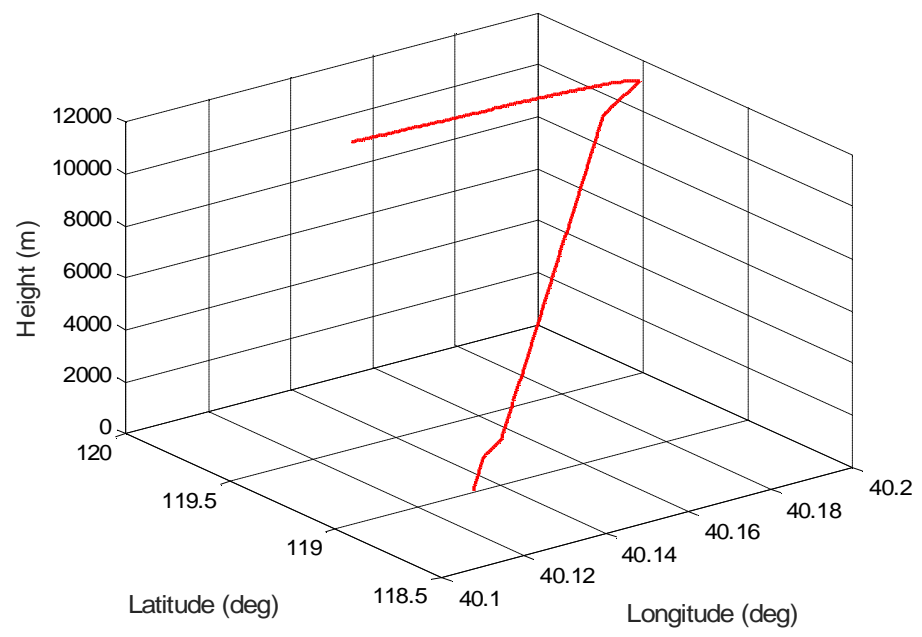

Fig. 1Reference trajectory of the aircraft

Fig. 2represents the position estimation errors of the MAFCKF and the CKF. It isobserved that the latitude error and the height error of the CKF both diverge, and the estimation errors of the MAFCKF are all fluctuating around zeros. The latitude and longitude estimation errors of the MAFCKF are mostly in $\pm 20 \mathrm{~m}$, and the height estimate errors of the MAFCKF are mostly in $\pm 50 \mathrm{~m}$. The velocity estimation errors of the above two filters are shown in Fig. 3. The errors of the CKF are all divergent at the end time. The velocity errors of the MAFCKF are mostly in $\pm 0.2 \mathrm{~m} / \mathrm{s}$. In simulations, the CKF has a bad performance to endure the drifts of the accelerometer, the uncertain of the dynamic covariance and measurement covariance, and the fast-maneuvering of the aircraft. The MACKFworks well because of introducing the multiple fading factors. These results demonstrate that the MACKF can effectively reduce the estimation errors in the presence of the uncertainties of the INS/GPS integrated navigation.
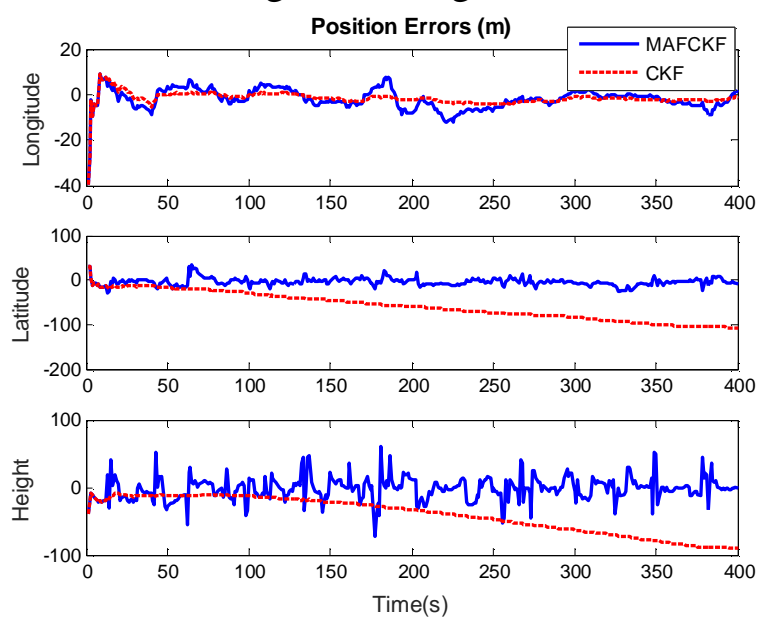

Fig. 2 Position errors of the MAFCKF and the $\mathrm{CKF}$
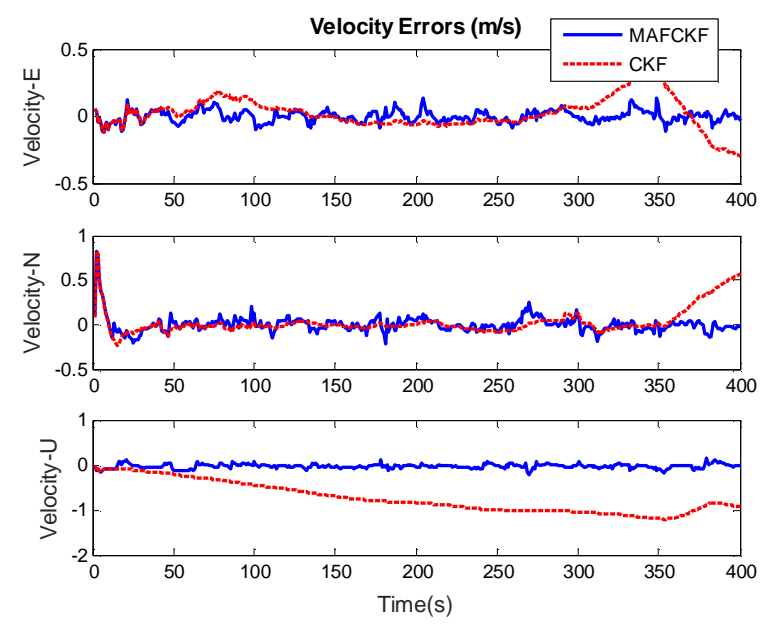

Fig. 3 Velocity errors of the MAFCKF and the $\mathrm{CKF}$ 


\section{Conclusions}

Amultiple adaptive fading cubature Kalman filter (MAFCKF)for integrated navigation system with inaccuratemodels is proposed. Firstly, the dynamics and measurement model of the INS/GPS integrated navigation system is introduced. Secondly, a multiple fadingfactors is designed by using co-covariance of the state and measurement, and the covariance is calculate by the propagation of thecubature points. Finally, simulations for theINS/GPS integrated navigation system by using the MAFCKF and the CKF are carried out, and the results of the integrated navigation are analyzed.

\section{References}

[1] J. L. Crassidis and J. L. Junkins, Optimal estimation of dynamic systems, 2nd ed. ed. vol. 24. Boca Raton, FL: Chapman \& HalllCRC Press, 2012.

[2] X. Y. Z. Z. Zhou DH, "A Suboptimal Multiple Fading Extended Kalman Filter_x000D_x000A_," Acta Automatica Sinica, vol. 17, pp. 689-695+758, 1991.

[3] Q. Xia, M. Rao, Y. Ying, and X. Shen, "Adaptive fading Kalman filter with an application," Automatica, vol. 30, pp. 1333-1338, 1994.

[4] C. Hide, "Adaptive Kalman Filtering for Low-cost INS/GPS," Journal of navigation, vol. 56, pp. 143-152, 2003.

[5] T. Lou, Z. Wang, M. Xiao, and H. Fu, "Multiple Adaptive Fading Schmidt-Kalman Filter for Unknown Bias," Mathematical Problems in Engineering, vol. 2014, p. 8, 2014.

[6] I. Arasaratnam and S. Haykin, "Cubature Kalman filters," Automatic Control, IEEE Transactions on, vol. 54, pp. 1254-1269, 2009.

[7] B. Jia, M. Xin and Y. Cheng, "High-degree cubature Kalman filter," Automatica, vol. 49, pp. 510-518, 2013.

[8] Y. Ho and R. Lee, "A Bayesian approach to problems in stochastic estimation and control," Automatic Control, IEEE Transactions on, vol. 9, pp. 333-339, 1964. 